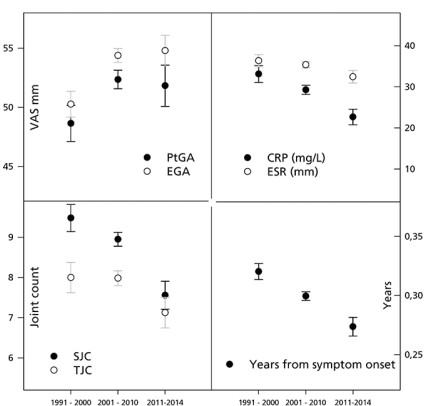

Figure 1. Mean baseline values with $95 \%$ confidence interval.

Disclosure of Interests: Jon Einarsson: None declared, Tor Olofsson: None declared, Olafur Palsson: None declared, Johan K Wallman Consultant for: Consultant for AbbVie, Celgene, Eli Lilly, Novartis, and UCB Pharma., Meliha C Kapetanovic: None declared DOI: 10.1136/annrheumdis-2019-eular.2167

\section{THU0074 EVALUATION OF MEDICATION PERSISTENCE IN PATIENTS WITH RHEUMATOID ARTHRITIS AND SJÖGREN'S SYNDROME/INTERSTITIAL LUNG DISEASE TREATED WITH NON-TNFI DISEASE-MODIFYING ANTI- RHEUMATIC DRUGS}

Leticia Ferri ${ }^{1}$, Evo Alemao ${ }^{1}$, Aarti Rao ${ }^{2} .{ }^{1}$ Bristol-Myers Squibb, Princeton, United States of America; ${ }^{2}$ Mu Sigma, Bangalore, India

Background: RA is a systemic disease driven by pathogenic autoantibodies and inflammatory cytokines that can involve tissues and organs as well as synovial joints. Sjögren's syndrome (SS) and interstitial lung disease (ILD) are frequently reported extra-articular manifestations of RA. However, there are currently limited data on the persistency of non-TNF inhibitor (TNFi) biologic (b)DMARDs and targeted synthetic DMARDs, such as tofacitinib (TOF), in patients (pts) with SS and ILD secondary to RA (RA-SS and RA-ILD).

Objectives: To compare medication persistence between non-TNFi DMARDs including abatacept (ABA), TOF and tocilizumab (TOC) in RASS and RA-ILD.

Methods: Pts ( $>18$ years) from the Truven MarketScan ${ }^{\mathrm{TM}}$ administrative claims database with $R A$ ( $\geq 2$ prescription claims for RA identified with International Classification of Diseases [ICD]-9 or ICD-10) being treated with a non-TNFi DMARD from Jan 2006 to Sep 2017 were included. The date of the first claim for a drug of interest on or after diagnosis of RA was considered the index date. Pts were required to have at least 6 months of continuous enrolment prior to the index date (baseline). Pts with claims for other bDMARDs on the index date were excluded from the study. Pts with a diagnosis of SS or ILD any time in the baseline or follow-up periods were included in the RA-SS or RA-ILD cohorts, respectively. Persistence was defined as the number of days from index date to first switch or discontinuation of the index date DMARD, or end of the study period, or end of enrolment, whichever came first. Pts in both cohorts were stratified by prior TNFi use (TNFi-naïve or TNFi-experienced) and by prior corticosteroid (CS) use. Pairwise comparison of persistence between the non-TNFi DMARDs was performed with ABA as the reference, using the Kruskal-Wallis test for both cohorts separately. A $p$ value of $<0.05$ was considered statistically significant.

Results: A total of 2503 and 3856 pts satisfied the inclusion and exclusion criteria for the RA-SS and RA-ILD cohorts, respectively. In the RASS cohort, 1767, 378 and 358 pts were taking ABA, TOF and TOC, respectively; in the RA-ILD cohort, these numbers were 2937, 343 and 576 . The majority ( $90 \%$ in RA-SS and $75 \%$ in RA-ILD) of the study population was female. In the RA-ILD cohort, the proportion of pts with prior CS use was significantly lower in pts taking ABA (vs TOF). In the RASS cohort, the comorbidity index between treatment cohorts was similar; however, ABA pts had significantly lower pulmonary nodules vs TOF pts (Table 1). In the RA-ILD cohort, the comorbidity index was significantly lower for ABA (vs TOF and TOC), with differences in types of comorbidities at baseline (Table 1). In the RA-SS cohort, the mean time on therapy for pts in the ABA cohort was longer compared with pts in the TOF cohort. Persistency of ABA was higher compared with TOF in the TNFiexperienced pts and in CS-experienced pts (Table 2). In the RA-ILD cohort, overall mean time on therapy for pts taking ABA was longer compared with TOC. Persistency of ABA was higher compared with TOC in the TNFi-naïve cohort.

Conclusion: Based on the analysis of a large US claims database, pts with RA-SS prescribed ABA (vs TOF) had higher persistency, and pts with RA-ILD prescribed ABA (vs TOC) had higher persistency. Further analysis of medication persistence, adjusting for pt characteristics, is warranted.

Abstract THU0074-Table 1. demographics

Table 1. Baseline characteristics by cohort

\begin{tabular}{|c|c|c|c|c|c|c|}
\hline & \multicolumn{3}{|c|}{ RA-SS cohort } & \multicolumn{3}{|c|}{ RA-ILD cohort } \\
\hline & $\underset{\substack{\mathrm{ABA} \\
(\mathrm{n}=1767)}}{2}$ & $\begin{array}{c}\text { TOF } \\
(n=378)\end{array}$ & $\begin{array}{c}\text { TOC } \\
(\mathrm{n}=358)\end{array}$ & $\begin{array}{c}\mathrm{ABA} \\
(\mathrm{n}=2937)\end{array}$ & $\begin{array}{c}\text { TOF } \\
(n=343)\end{array}$ & $\begin{array}{c}\text { TOC } \\
(n=576)\end{array}$ \\
\hline Mean (SD) age, years & $55.1(11.4)$ & $54.6(10.5)$ & $55.6(12.1)$ & $61.4(12.0)$ & $62.4(11.3)$ & \\
\hline Female, $\mathbf{n}(\%)$ & $1635(92.5)$ & $349(92.3)$ & $325(90.8)$ & $2247(76.5)$ & $262(76.4)$ & $449(78.0)$ \\
\hline $\begin{array}{l}\text { TNFi-experienced, } \mathbf{n} \\
(\%)\end{array}$ & $829(46.9)$ & $175(46.3)$ & $213(59.5)^{*}$ & $1338(45.6)$ & $132(38.5)^{*}$ & $297(51.6)^{*}$ \\
\hline CS use, $\mathrm{n}(\%)$ & $1114(63.0)$ & $257(68.0)$ & $221(61.7)$ & $1885(64.2)$ & $261(76.1)^{*}$ & $375(65.1)$ \\
\hline $\mathrm{CCl}$ score, mean (SD) & $1.4(0.9)$ & $1.4(0.9)$ & $1.5(0.9)$ & $1.8(1.2)$ & $2.0(1.3)^{*}$ & $1.9(1.4)^{*}$ \\
\hline \multicolumn{7}{|c|}{ Select comorbidities, $n(\%)$} \\
\hline COPD/asthma & $19(1.1)$ & $7(1.9)$ & $6(1.7)$ & $118(4.0)$ & $39(11.4)^{*}$ & $40(6.9)^{2}$ \\
\hline Pneumonia & $60(3.4)$ & $19(5.0)$ & $16(4.5)$ & $309(10.5)$ & $53(15.5)^{x}$ & $68(11.8)$ \\
\hline Pulmonary nodule & $47(2.7)$ & $22(5.8)^{*}$ & $12(3.4)$ & $216(7.4)$ & $81(23.6)^{x}$ & $89(15.5)^{*}$ \\
\hline Heart failure & $42(2.4)$ & $3(0.8)$ & $5(1.4)$ & $219(7.5)$ & $31(9.0)$ & $45(7.8)$ \\
\hline Dyslipidaemia & $269(15.2)$ & $66(17.5)$ & $67(18.7)$ & $526(17.9)$ & $104(30.3)^{x}$ & $138(24.0)^{x}$ \\
\hline $\begin{array}{l}\text { Ischemic heart } \\
\text { disease }\end{array}$ & $100(5.7)$ & $19(5.0)$ & $22(6.1)$ & $418(14.2)$ & $70(20.4)^{x}$ & $68(11.8)$ \\
\hline Osteoarthritis & $524(2$ & $126(33.3)$ & $118(33.0)$ & $935(31.8)$ & $127(37)$ & $209(36.3)^{*}$ \\
\hline Lupus & 171(9.7) & $26(6.9)$ & $37(10.3)$ & $132(4.5)$ & $9(2.6)$ & $28(4.9)$ \\
\hline Infections & $953(53.9)$ & $198(52.4)$ & $187(52.2)$ & $1613(54.9)$ & $192(56.0)$ & $330(57.3)$ \\
\hline
\end{tabular}

Abstract THU0074-Table 2. demographics

Table 2. Persistence by cohort: overall and stratified by prior TNFi and $\mathrm{CS}$ us

\begin{tabular}{|c|c|c|c|c|c|c|}
\hline & \multicolumn{3}{|c|}{ RA-SS cohort } & \multicolumn{3}{|c|}{ RA-ILD cohort } \\
\hline & ABA $(n=1767)$ & TOF $(n=378)$ & TOC $(n=358)$ & ABA $(n=2937)$ & TOF $(n=343)$ & TOC $(n=576)$ \\
\hline Overall & $\begin{array}{c}\mathrm{n}=1767 \\
367.9(440.5) \\
204\end{array}$ & $\begin{array}{c}n=378 \\
281.2(310.3)^{*} \\
167\end{array}$ & $\begin{array}{c}\mathrm{n}=358 \\
316.1(343.6) \\
175\end{array}$ & $\begin{array}{c}\mathrm{n}=2937 \\
367.4(439.9) \\
211\end{array}$ & $\begin{array}{c}\mathrm{n}=343 \\
315.3(317.9) \\
186\end{array}$ & $\begin{array}{c}n=576 \\
316.0(366.9)^{*} \\
179\end{array}$ \\
\hline TNFi-naive & $\begin{array}{c}\mathrm{n}=938 \\
357.9(447.8) \\
193\end{array}$ & $\begin{array}{c}n=203 \\
269.6(281.9) \\
178\end{array}$ & $\begin{array}{c}\mathrm{n}=145 \\
297.5(374.6) \\
147\end{array}$ & $\begin{array}{c}n=1599 \\
358.5(426.5) \\
201\end{array}$ & $\begin{array}{c}\mathrm{n}=211 \\
307.3(317.3) \\
165\end{array}$ & $\begin{array}{c}n=279 \\
269.7(311.6)^{*} \\
156\end{array}$ \\
\hline $\begin{array}{l}\text { TNFi- } \\
\text { experienced }\end{array}$ & $\begin{array}{c}\mathrm{n}=829 \\
379.1(432) \\
225\end{array}$ & $\begin{array}{c}\mathrm{n}=175 \\
294.7(340.5)^{*} \\
163\end{array}$ & $\begin{array}{c}\mathrm{n}=213 \\
328.7(321.1) \\
205\end{array}$ & $\begin{array}{c}\mathrm{n}=1338 \\
378(455.3) \\
223\end{array}$ & $\begin{array}{c}\mathrm{n}=132 \\
328.3(319.5) \\
211.5\end{array}$ & $\begin{array}{c}n=297 \\
359.6(408) \\
208\end{array}$ \\
\hline CS-naive & $\begin{array}{c}\mathrm{n}=653 \\
368.4(441.7) \\
197\end{array}$ & $\begin{array}{c}n=121 \\
327.2(313.6) \\
235\end{array}$ & $\begin{array}{c}\mathrm{n}=137 \\
311.2(351.5) \\
175\end{array}$ & $\begin{array}{c}\mathrm{n}=1052 \\
360.7(443.7) \\
208\end{array}$ & $\begin{array}{c}\mathrm{n}=82 \\
363.1(322.8) \\
281\end{array}$ & $\begin{array}{c}\mathrm{n}=201 \\
303.9(357.4) \\
175\end{array}$ \\
\hline $\begin{array}{l}\text { CS- } \\
\text { experienced }\end{array}$ & $\begin{array}{c}\mathrm{n}=1114 \\
367.6(440) \\
206\end{array}$ & $\begin{array}{c}\mathrm{n}=257 \\
259.6(306.9)^{*} \\
138\end{array}$ & $\begin{array}{c}\mathrm{n}=221 \\
319.1(339.4) \\
190\end{array}$ & $\begin{array}{c}\mathrm{n}=1885 \\
371.1(437.8) \\
213\end{array}$ & $\begin{array}{c}\mathrm{n}=261 \\
300.3(315.4)^{*} \\
164\end{array}$ & $\begin{array}{c}\mathrm{n}=375 \\
322.6(372.2)^{*} \\
181\end{array}$ \\
\hline
\end{tabular}

Disclosure of Interests: Leticia Ferri Shareholder of: Bristol-Myers Squibb, Employee of: Bristol-Myers Squibb, Evo Alemao Shareholder of: BristolMyers Squibb, Employee of: Bristol-Myers Squibb, Aarti Rao Consultant for: Bristol-Myers Squibb

DOI: 10.1136/annrheumdis-2019-eular.1480

\section{THU0075 EARLY VERSUS DELAYED START OF BARICITINIB IN PATIENTS WITH RHEUMATOID ARTHRITIS IN A PHASE 3 TRIAL OF PATIENTS NAÏVE TO METHOTREXATE TREATMENT}

Roy Fleischmann ${ }^{1}$, Michael Schiff ${ }^{2}$, Tore K. Kvien ${ }^{3}$, Cem Gabay $^{4}$, Patrick Durez ${ }^{5}$ Anabela Cardoso $^{6}$, Jinglin Zhong ${ }^{7}$, Yun-Fei Chen ${ }^{6}$, Jennifer Workman ${ }^{6}$, Tsutomu Takeuchi ${ }^{8} .{ }^{1}$ University of Texas Southwestern Medical Center, Dallas, United States of America; ${ }^{2}$ University of Colorado, Denver, United States of America; ${ }^{3}$ Diakonhjemmet Hospital, Oslo, Norway; ${ }^{4}$ University of Geneva, Geneva, Switzerland; ${ }^{5}$ UCL-Saint Luc, Bruxelles, Belgium; ${ }^{6}$ Eli Lilly and Company, Indianapolis, United States of America; ${ }^{7} I Q V I A$, Morrisville, United States of America; ${ }^{8}$ Keio University School of Medicine, Tokyo, Japan

Background: Baricitinib (bari) is an oral Janus kinase (JAK)1/JAK2 inhibitor approved to treat moderately to severely active rheumatoid arthritis (RA) in adults in over 50 countries including European countries, the US and Japan. In the 52-week (wk) Phase 3 RA-BEGIN study, bari 4-mg alone or in combination with methotrexate (MTX) showed clinical improvements compared to MTX monotherapy for MTX-naïve patients (pts) with early active RA.

Objectives: To assess if pts who receive bari monotherapy early attain enhanced clinical, functional and radiographic outcomes compared to pts who initiated MTX and switched to bari at wk 52 . 
Methods: In RA-BEGIN, 588 pts (mean disease duration 1.4 years) were randomized 4:3:4 to MTX, bari 4-mg once-daily, or combination MTX and bari 4-mg. At Wk 52, pts could enter a long-term extension study in which all pts received open-label bari 4-mg. Pts initially randomized to bari-4 mg monotherapy were defined as early-start and MTX pts who switched to bari 4-mg at Wk 52 were defined as delayed-start for this analysis. Change from baseline using mixed model repeated measures and mean scores were assessed for the Simplified Disease Activity Index (SDAI), Clinical Disease Activity Index (CDAI), Disease Activity Score 28joints-high-sensitivity C-reactive protein (DAS28-hsCRP), Disease Activity Score 28-joints-erythrocyte sedimentation rate (DAS28-ESR), Health Assessment Questionnaire-Disability Index (HAQ-DI), and modified Total Sharp Score (mTSS) to compare early- vs delayed-start groups between Wks 0 and 100 (Wks 52-100 for mTSS). Percent of pts achieving low disease activity (LDA, SDAI $\leq 11$ ) and remission (SDAI $\leq 3.3$ ) were also assessed.

Results: LDA and remission response rates in the delayed-start group increased from $60 \%$ to $78 \%$ and $18 \%$ to $31 \%$, respectively, within 4 wks after switching to bari $4-\mathrm{mg}$. Remission rates reached $47 \%$ within 1 year after switching, but were not different from the group that initiated bari (Figure 1). Similar results were seen for CDAI, DAS28-ESR, and DAS28hsCRP (only up to Wk 40 for DAS28-ESR) (data not shown). Pts initially randomized to bari 4-mg had significantly greater change from baseline, observed as early as $W k 1$ and up to Wk 52, for HAQ-DI than pts treated with MTX (Figure 2). After switching to bari, the delayed-start group showed a rapid improvement in HAQ-DI with similar improvement at Wk 56 - 4 wks after switching to bari. Upon initiating bari, there was a reduction in the rate of structural damage; however, delaying initiation of bari for 1 year resulted in numerically higher mTSS

Conclusion: In early MTX-naïve pts, improved clinical, functional, and radiographic efficacy with bari 4-mg vs MTX was observed in most outcome measures up to 52 wks. Switching from MTX to bari at Wk 52 provided a rapid clinical response, allowing the majority of pts to achieve similar results 4 wks post-switch. However, if the goal of therapy is to achieve rapid and sustained control of disease activity, the differences noted here for HAQ-DI and especially structural progression support earlier switch to bari for pts who do not obtain disease control with MTX. Further analysis may be needed to explore prognostic factors, such as hsCRP and disease duration (< or $>6$ months), baseline erosion, or anticitrullinated protein antibody positivity, which may help clinicians identify pts who may benefit from earlier treatment with bari

\section{REFERENCES :}

[1] Fleischmann, et al. Arthritis \& Rheumatol. 2017;69:506-517

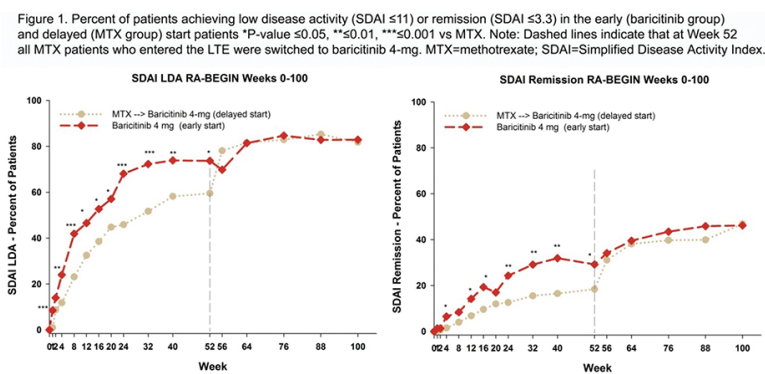

Abstract THU0075 - Figure 1

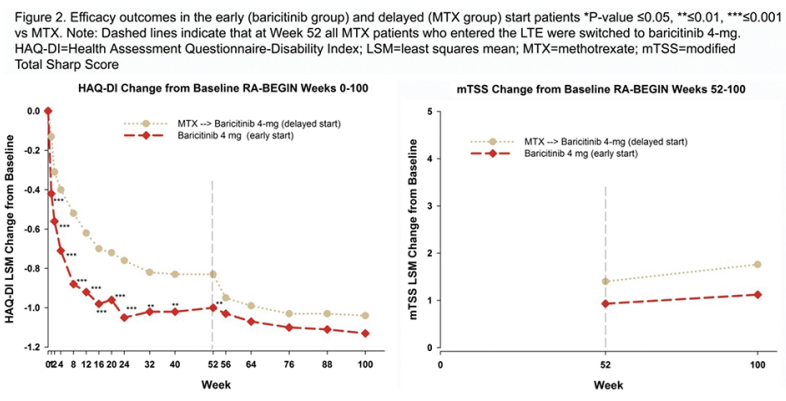

Abstract THU0075 - Figure 2
Disclosure of Interests: Roy Fleischmann Grant/research support from: AbbVie, Amgen, AstraZeneca, Bristol-Myers Squibb, Celtrion, Genentech, GSK, Janssen, Lilly, Novartis, Pfizer Inc, Sanofi-Aventis, UCB, Consultant for: AbbVie, Amgen, AstraZeneca, Bristol-Myers Squibb, Celtrion, Genentech, GSK, Janssen, Lilly, Novartis, Pfizer Inc, Sanofi-Aventis, UCB Michael Schiff Consultant for: Abbvie, BMS, Eli Lilly and Company, JJ, UCB, Speakers bureau: Abbvie, BMS, Tore K. Kvien Grant/research sup port from: AbbVie, BMS, MSD, Pfizer, Roche and UCB., Consultant for: AbbVie, Biogen, BMS, Boehringer Ingelheim, Celgene, Celltrion, Eli Lilly, Hospira, Merck-Serono, MSD, Novartis, Oktal, Orion Pharma, Pfizer, Roche, Sandoz, Sanofi, Mylan and UCB, Speakers bureau: AbbVie, Biogen, BMS, Boehringer Ingelheim, Celgene, Celltrion, Eli Lilly, Hospira, Merck-Serono, MSD, Novartis, Oktal, Orion Pharma, Pfizer, Roche, Sandoz, Sanofi and UCB, Cem Gabay Grant/research support from: Roche Pfizer, AB2 Bio Ltd, Consultant for: Roche, Pfizer, Lilly, AbbVie, Sanofi, Regeneron, Bristol-Myers Squibb, Novartis, UCB, AB2 Bio Ltd, Debiopharm, Patrick Durez Speakers bureau: Bristol-Myers Squibb, Eli Lilly, Sanofi, Celltrion, Anabela Cardoso Shareholder of: Eli Lilly and Company, Employee of: Eli Lilly and Company, Jinglin Zhong Shareholder of: El Lilly and Company, Employee of: Eli Lilly and Company, Yun-Fei Chen Shareholder of: Eli Lilly and Company, Employee of: Eli Lilly and Company, Jennifer Workman Shareholder of: Eli Lilly and Company, Employee of: Eli Lilly and Company, Tsutomu Takeuchi Grant/research suppor from: Astellas Pharma Inc, Chugai Pharmaceutical Co, Ltd., Daiichi Sankyo Co., Ltd., Takeda Pharmaceutical Co., Ltd., AbbVie GK, Asahikasei Pharma Corp., Mitsubishi Tanabe Pharma Co., Pfizer Japan Inc., Eisa Co., Ltd., AYUMI Pharmaceutical Corporation, Nipponkayaku Co. Ltd. Novartis Pharma K.K., Grant/research support from: AbbVie, Asahi Kasei, Astellas, AstraZeneca, AYUMI, Bristol-Myers Squibb, Chugai, Daiichi Sankyo, Eisai, Eli Lilly Japan, Janssen, Mitsubishi Tanabe, Nippon Kayaku, Novartis, Pfizer Japan Inc, Taiho, Taisho Toyama, Takeda, Teijin, Grant/ research support from: Astellas Pharma Inc., Bristol Myers Squibb, Chuga Pharmaceutical Co., Ltd., Mitsubishi Tanabe Pharma Co., Pfizer Japan Inc., Santen Pharmaceutical Co., Ltd., Takeda Pharmaceutical Co., Ltd., Teijin Pharma Ltd., AbbVie GK, Asahi Kasei Pharma Corp., Taisho Toyama Pharmaceutical Co., Ltd., SymBio Pharmaceuticals Ltd., Janssen Pharmaceutical K.K., Celltrion Inc., Nipponkayaku Co. Ltd., and UCB Japan, Consultant for: Astra Zeneca K.K., Eli Lilly Japan K.K., Novartis Pharma K.K., Mitsubishi Tanabe Pharma Co., Abbivie GK, Nipponkayaku Co.Ltd, Janssen Pharmaceutical K.K., Astellas Pharma Inc., Taiho Pharmaceutical Co. Ltd., Chugai Pharmaceutical Co. Ltd., Taisho Toyama Pharmaceutical Co. Ltd., GlaxoSmithKline K.K., UCB Japan Co. Ltd., Consultant for: AbbVie, Asahi Kasei, Astellas, AstraZeneca, AYUMI, Bristol-Myers Squibb, Chugai, Daiichi Sankyo, Eisai, Eli Lilly Japan, Janssen, Mitsubishi Tanabe, Nippon Kayaku, Novartis, Pfizer Japan Inc, Taiho, Taisho Toyama, Takeda, Teijin, Consultant for: Astra Zeneca K.K., El Lilly Japan K.K., Novartis Pharma K.K., Mitsubishi Tanabe Pharma Co., Asahi Kasei Medical K.K., AbbVie GK, Daiichi Sankyo Co., Ltd., Bristol Myers Squibb, and Nipponkayaku Co. Ltd., Speakers bureau: Astellas Pharma Inc., Bristol Myers Squibb, Chugai Pharmaceutical Co., Ltd., Mitsubishi Tanabe Pharma Co., Pfizer Japan Inc., Santen Pharmaceutical Co., Ltd., Takeda Pharmaceutical Co., Ltd., Teijin Pharma Ltd., AbbVie GK, Asahi Kasei Pharma Corp., Taisho Toyama Pharmaceutical Co., Ltd., SymBio Pharmaceuticals Ltd., Janssen Pharmaceutical K.K., Celltrion Inc. Nipponkayaku Co. Ltd., and UCB Japan, Speakers bureau: AbbVie, Asahi Kasei, Astellas, AstraZeneca, AYUMI, Bristol-Myers Squibb, Chugai, Daiichi Sankyo, Eisai, Eli Lilly Japan, Janssen, Mitsubishi Tanabe, Nippon Kayaku, Novartis, Pfizer Japan Inc, Taiho, Taisho Toyama, Takeda, Teijin, Speakers bureau: AbbVie GK., Bristol-Myers K.K., Chugai Pharmaceutical Co. Ltd., Mitsubishi Tanabe Pharma Co., Pfizer Japan Inc., Astellas Pharma Inc, Diaichi Sankyo Co. Ltd., Eisai Co. Ltd., Sanofi K.K., Teijin Pharma Ltd., Takeda Pharmaceutical Co. Ltd., Novartis Pharma K.K. DOI: 10.1136/annrheumdis-2019-eular.975

\section{THU0076 THE IMPACT OF VARYING EXCLUSION CRITERIA ON TREATMENT RESPONSE: REAL-WORLD EVIDENCE TO GUIDE THE DESIGN OF FUTURE CLINICAL TRIALS}

Thomas Frisell ${ }^{1}$, Scott Jelinsky ${ }^{2}$, Mark Peterson ${ }^{2}$, Johan Askling ${ }^{1} .{ }^{1}$ Karolinska Institutet, Solna, Sweden; ${ }^{2}$ Pfizer Research Technology Center, Cambridge, United States of America

Background: Clinical trials of novel pharmaceuticals in Rheumatoid Arthritis routinely use strict inclusion/exclusion criteria aimed at selecting a defined homogeneous population and producing a non-confounded demonstration of the agent's clinically relevant pharmacologic effect. However the act of strictly defining numerous individual criteria has the potential 